Z Rheumatol 2011 · 70:462-463

DOI 10.1007/s00393-011-0759-9

(c) Springer-Verlag 2011

\author{
E. Reinhold-Keller ${ }^{1} \cdot$ A. Zink ${ }^{2}$ \\ ${ }^{1}$ Internistisch-rheumatologische Gemeinschaftspraxis, Hamburg \\ 2 Programmbereich Epidemiologie, Deutsches Rheuma-Forschungszentrum Berlin, \\ ein Leibniz-Institut, Berlin
}

\title{
Morbidität und Mortalität bei entzündlich-rheumatischen Krankheiten: Wie haben sie sich verändert?
}

pus erythematodes (SLE) und Vaskulitiden verändert haben.

Bei der RA ist das gegenüber der Bevölkerung erhöhte Mortalitätsrisiko v. a. durch die höhere kardiovaskuläre Morbidität bedingt. Kleinert und Krüger belegen aufgrund einer Vielzahl von Studien, dass die krankheitsbedingte Entzündung ein erhebliches Risiko für kardiovaskuläre Ereignisse bedeutet, zusätzlich zu und unabhängig von traditionellen Risikofaktoren. Die erfolgreiche Kontrolle der Krankheitsaktivität senkt damit auch das Mortalitätsrisiko. Erste Ergebnisse zeigen eine Verbesserung der Lebenserwartung in jüngerer Zeit.

Bei den Spondyloarthritiden stehen Schmerzen und eingeschränkte Funktion durch Entzündung und Versteifung des Achsenskeletts sowie durch periphere Gelenkbeteiligung im Vordergrund. Bei schweren Verlaufsformen ist die Gesamtmortalität um etwa 50\% erhöht. Auch bei diesen Krankheitsbildern ist mit einer erhöhten kardiovaskulären Morbidität zu rechnen, die einer gezielten Intervention bedarf. Dies ist umso sinnvoller, als beispielsweise das Rauchen nicht nur das kardiovaskuläre Risiko erhöht, sondern auch mit einem schwereren Krankheitsverlauf assoziiert ist.

Entzündlich-rheumatische Systemerkrankungen - in 2 weiteren Beiträgen am Beispiel des SLE und den ANCA-assoziierten Vaskulitiden (AAV) dargestellt - stellen aufgrund ihrer facettenreichen klinischen Bilder immer eine besondere Herausforderung im klinischen Alltag dar. Aufgrund ihrer in aller Regel vorhandenen Multiorganmanifestationen erfordern sie generell eine interdisziplinäre Betreuung. Beide Beiträge zu diesen Krankheitsbildern in diesem Heft dokumentieren für diese Krankheitsgruppen in den letzten Jahrzehnten eine deutlich rückläufige Mortalität, gemessen an der ,standardized mortality rate" (SMR) im Vergleich zur Normalbevölkerung. Bei den AAV können heute einzelne „subsets“ durchaus mit einer normalen Lebenserwartung rechnen. Dazu hat bei den AAV neben der deutlich früheren Diagnose v. a. das aktivitäts- und stadienadaptierte therapeutische Vorgehen geführt, das im letzten Jahrzehnt für die meisten Patienten auf der Grundlage von prospektiven kontrollierten multizentrischen Studien erfolgte.

In der Frühmortalität spielen nach wie vor bei beiden Krankheitsgruppen Infektionen die Hauptrolle durch die in aller Regel initial am stärksten immunsuppressive Therapie. Aufgrund der insgesamt aber deutlich verbesserten Prognose rücken auch beim SLE und den AAV krankheits- und therapiebedingte Komorbiditäten im Langzeitverlauf in den Vordergrund. Für beide Erkrankungen spielt hier die permanente Nierenfunktionseinschränkung eine besondere Rolle; sie bedingt eine deutlich erhöhte Mortalität. Besonderes Augenmerk bei AAV-Patienten erfordern die Folgen der früher oft mehrjährig durchgeführten Cyclophosphamidtherapie. Wie der Beitrag zu den AAV zeigt, ist zwar auch heute Cyclophosphamid noch unverzichtbarer Therapie- 
bestandteil bei den meisten Patienten mit schweren lebens- oder organbedrohenden Verläufen, jedoch wird es heute wesentlich kürzer mit deutlich reduzierten Kumulativdosen eingesetzt, sodass diese Therapiefolgen in Zukunft nur noch von untergeordneter Bedeutung sein dürften.

Während für SLE-Patienten bereits zahlreiche Untersuchungen $\mathrm{zu}$ einem deutlich erhöhten kardiovaskulären Risiko als eine Haupttodesursache im Spätverlauf vorliegen, ist die Datenlage für die AAV dazu noch spärlich. Aber es deutet sich auch hier ein deutlich erhöhtes Risiko für Herz-Kreislauf-Erkrankungen an.

Für alle in diesem Schwerpunktheft vorgestellten Erkrankungen kann heute eine deutlich verbesserte Prognose hinsichtlich Mortalität und Morbidität dokumentiert werden. Zunehmend rücken jetzt Komorbiditäten in den Fokus des Interesses, insbesondere das für alle Krankheiten belegte erhöhte kardiovaskuläre Risiko. Obwohl kontrollierte Interventionsstudien bislang fehlen, sollten neben einer konsequenten Therapie der Grundkrankheit von Anfang an kardiovaskuläre Risikofaktoren beachtet und in das Behandlungskonzept einbezogen werden.

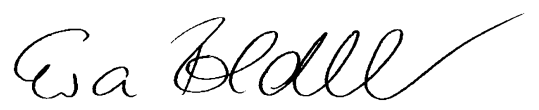

PD Dr. Eva Reinhold-Keller, Hamburg

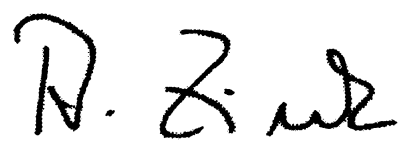

Prof. Dr. Angela Zink, Berlin

\section{Korrespondenzadressen}

\section{PD Dr. E. Reinhold-Keller}

Internistisch-rheumatologische Gemeinschaftspraxis

Jürgensallee 44, 22609 Hamburg

reinhold-keller@rheuma-spezialisten.de

\section{Prof. Dr. A. Zink}

Programmbereich Epidemiologie,

Deutsches Rheuma-Forschungszentrum

Berlin, ein Leibniz-Institut

Charitéplatz 1, 10117 Berlin

zink@drfz.de

\title{
Die Suche nach dem Heiligen Gral: Kosten-Nutzen-Bewertung in der internistischen Krankenbetreuung
}

\author{
Versorgungsforschung und GKV-Leistungs- \\ katalog einbeziehen. Eine breitere Öffentlichkeit \\ in die Debatte einbeziehen
}

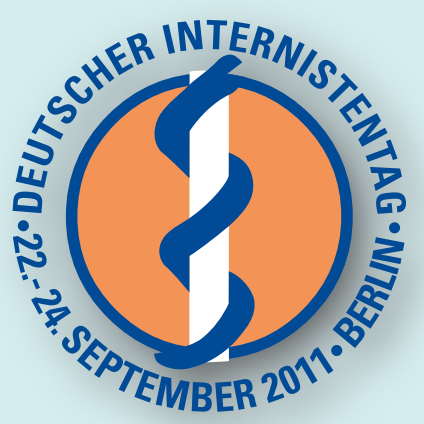

Auch nach dem Ende der Amtszeit von Jörg-Dietrich Hoppe, der sich in der sogenannten „Priorisierungsdebatte" stark engagiert hatte, bleibt das Thema auf der Agenda: die Notwendigkeit, medizinische Leistungen nach ihrem Kosten und Nutzen zu bewerten. Die medizinische Forschung schreitet voran. Immer mehr Behandlungsmethoden stehen zur Verfügung. Gleichzeitig schwinden die nötigen finanziellen Rücklagen im Versichertensystem. Die Frage nach dem Umgang mit den knappen Mitteln im Gesundheitswesen spitzt sich zu: Mit welchen Instrumenten kann dieser Mittelknappheit begegnet werden? Welche politischen Schritte stehen an?

Dr. Wesiack, Präsident des Berufsverbandes Deutscher Internisten (BDI), nimmt die Politik in die Pflicht: „Vom Gesetzgeber wird das Problem der Mittelknappheit und die damit einhergehende Notwendigkeit zur Rationalisierung bisher standhaft ignoriert", so Wesiack. „Dabei mehren sich die Anzeichen für Qualitätseinbußen aufgrund begrenzter Ressourcen“, fügt er hinzu. „Wir brauchen ein funktionierendes Werkzeug zur Kostenund Nutzenbewertung. Hierfür muss die Versorgungsforschung ausgebaut und der Leistungskatalog der GKV neu bestimmt werden." Die Diskussion müsse über eine reine Fokussierung auf Rationalisierungsmodelle hinausgehen. Der BDI plädiert außerdem dafür, den bisher überwiegend wissenschaftlich geführten Diskurs auf eine breitere öffentliche Ebene zu bringen. Denn entgegen dem mancherorts heraufbeschworenen Anschein gibt es ein objektives Bewertungsinstrument für medizinische Leistungen bisher nicht. Ökonomische Verfahren zur Definition, Bewertung und Umsetzung der Begriffe "Kosten" und "Nutzen" allein reichten hierfür nicht aus. Ein funktionierendes Instrument zur KostenNutzen-Bewertung könne darum nur über eine breiter angelegte Auseinandersetzung mit der Thematik erreicht werden.

Zu diesem Zweck ruft der BDI auf dem 4. Deutschen Internistentag in Berlin ein qualifiziert besetztes Podium zusammen: Ulrike Flach, Parlamentarische Staatssekretärin im Bundesministerium für Gesundheit, Dr. med. Stefan Etgeton, Verbraucherzentrale Berlin, Dr. jur. Rainer Hess, Vorsitzender Gemeinsamer Bundesausschuss (G-BA), Dr. Günther Jonitz, Präsident der LÄK Berlin und Prof. Dr. Herbert Rebscher, Vorstandsvorsitzender DAK, erörtern gemeinsam die Problematik.

\section{- Veranstaltung}

"Kosten- und Nutzenbewertung in der internistischen Krankenbetreuung" am Do., 22. September 2011 um 15.15 Uhr im RAMADA Hotel, Berlin-Alexanderplatz

Mehr unter: www.internistentag.de 